\title{
Advanced Booking Discount Program: A Coordinating Strategy for SMEs Food Processors on Managing Demand Uncertainty
}

\author{
Juma Makweba Ruteri (Corresponding author) \& Qi Xu \\ Glorious School of Business and Management, Donghua University \\ PO box 474, 1882 West Yan an Rd Shanghai, China \\ Tel: 86-2-16237-3965Ｅ-mail: jmakweba@yahoo.com
}

\begin{abstract}
Because demand for processed food products is uncertain, the processors face dilemma to satisfy their customers. The processors are unable to sell their products if demand turns out to be low. That is, some of the products will expire, the producer would have been better off by producing less. But if the processors choose to produce less to ensure that the entire products are sold, there will be an excess of demand if demand turns out to be high. In this case the processors would have been better off by choosing to produce large quantity. The dilemma is more experienced by SMEs food processors because they lack enough capital to acquire advanced technologies to assist in decision making. In this paper an attempt has been made to address the coordinating effects of ABD program in managing demand uncertainty. Fruits and vegetables drying factory has been focused as a case study. Theoretical expressions supported by explanatory mathematical equations have been offered. It is noted that the processor may achieve better decisions and managed demand uncertainty once they agree upon information sharing.
\end{abstract}

Keywords: Advanced booking discount, Supply chain coordination, Production decision, Demand uncertainty, SMEs

\section{Introduction}

Challenges in the food industry sector regardless of the size (small or large) are diverse and demanding, and need to be addressed on several fronts to improve service levels and take unnecessary inventory out of the supply chain and achieve higher customer service levels while meeting local and international regulations. Coordination mechanisms provide a system for a supply chain partners to collectively create value and achieve improved supply chain performance.

A supply chain is believed to be coordinated if the decisions the retailer and the processor make maximizes the total supply chain profits. In reality, each stage in a supply chain operate independently and attempt to maximize individual profit. The result of this independent decision making is lack of coordination because actions that maximize retailer's profit may not necessarily maximize the processor's profit. In our view, chain performance in the food processing industries is hindered by the presence of uncertainties to do with decision making. The uncertainty observed frequently in the food industries such as deliveries, product recalls, demand fluctuation, consumers' perceptions, machine breakdowns, and lead times leads to increased customers' dissatisfactions. Efficient and effective supply chain strategies are therefore needed to reduce those uncertainties not only in the entire supply chain of large food processing industries but also in small and medium enterprises (SMEs).

Processed food products have high unpredictable demand. It is a trend that demand is low during harvest season as most of people prefer to eat fresh harvested fruits and vegetables or other raw materials rather than consuming processed products. Similarly for drinks, demand is low during cold weather and significant increase during hot season. For the processor is more cost effective to buy and produce different products during harvest season as the price of raw materials become low. SMEs experience more pressure on handling freshly harvested materials as they are perishable. Despite of available advanced technologies around the world, most of SMEs food processors can not afford to buy these technologies because of capital constraint. In steady SMEs opt to use poor and labor intensive technologies with low production capacities accompanied with low skilled and less experienced personnel. To provide a high level of product availability it requires production of large quantity of products. High level of product availability is likely to satisfy unexpected demand, but also is likely to result in a large quantity of expired products at the end of the selling season. Low product availability is likely to result in few expired products, but also it is quite clear that other customers willing to buy will turn away without the product because of stock out. Thus, responding accurately to changing 
demand patterns by forecasting and translating forecasts into an efficient production and distribution plan are key ingredients for success.

The majority of food processors use push supply model where by the product goes through the distribution network without orders in hand, in such strategy production and inventory held are therefore guided by predictions or anticipation of customer demand. Financial and technological constraints impose difficulties for SMEs to employ just-in-time (JIT) approach or pull supply model; the supply chain strategies which production process focus on customer needs. The strategies help to minimize inventory and avoid the risk of excess production of unwanted products. On the other hand, customers are not willing to wait the time it would take to process their order through the supply chain and make the product based on their order.

Working as an individual entity it becomes very difficult to attain supply chain efficiency as the food processing sector encounter a number of uncertainties which need joint efforts to address. Inspired by Tang et al. (2002) findings, this paper presents and analyses the impact of advanced booking discount (ABD) program as a coordinating strategy for SMEs food processors in managing demand uncertainty. Fruits and vegetables drying factory is referred to as a case study to demonstrate the coordinating effects of ABD program. In this study we consider that the retailer offer the program as a selling strategy to attract customers to buy more.

\section{Literature review}

In this section, selected works on supply chain strategies are reviewed. Having reviewed the literature two strategies for the purpose of this study have been identified as one of the strategies reported to improve supply chain performance as well as profit maximization. Hence, the review is based on two factors; discounts and coordination. Furthermore, we extended our review on demand uncertainty and some characteristics of SMEs. The objective of this review is to ascertain the correlation mechanisms that can help to develop theoretical description in managing demand uncertainty.

\subsection{Discounts}

Provision of discounts to entice customers in different business sectors has been widely used. For the manufacturer discounts induce the wholesaler or retailer to order more while for the retailer discounts entice consumers to purchase more. A number of literatures and models addressing different discount schemes are also widely available as well. Lau, et al. (2008) presented quantity discount and handling charge for a manufacturer supplying numerous heterogeneous retailers. In the model, they showed a "price break" referred to the order size needed to qualify for quantity discount or handling charge reduction. Schotanus et al. (2009) studied an analytical and empirical basis for one general quantity discount function that can be used to describe the underlying function of almost all different quantity discount types. For a broad literature review on quantity discount few to mention, the reader is referred to (Crama et al. 2004, $\mathrm{Li}$ and Liu 2006, Lau et al. 2007, Burke et al. 2008, Mirmohammadi et al. 2009). Tang et al. (2002) discussed an alternative strategy on improving demand accuracy forecast referred to as advanced booking discount program. The essence of this program is to overcome the difficulties in responding accurately to changing demand especially for products with short life cycles and high demand uncertainty. The program attracts the customers to pre-commit and pay their orders at a discount price before the selling season. However, once the customer commits and pay, no cancellation or refund is allowed and the order is delivered during the selling season. Purchase in advance under non-refundable discount price and redeem the service in a later period has been widely pronounced in the service industry sector such as in the hotels, airlines, tourist companies among others (Lee and $\mathrm{Ng}$ 2001, Schwartz 2006) . In the food industry Maxim's bakery in Hong Kong provide a successful story on the use of ABD program for the sales of moon cakes a Chinese traditional cake consumed during mid-Autumn festival. The bakery offer $25 \%$ off the regular price, while the customers who do not pre-commit their orders, they buy the cake at the regular selling price.

Lee and $\mathrm{Ng}$ (2001) have shown theoretically that when the firm undertakes advanced sale, capacity utilization and profits are higher even though prices for sale in advance are discounted. The firm can offer advanced sell for cash flow reasons, to maximize cash revenues, to preempt competition for an alternative product, to ease planning and budget based on committed revenues. Perishability also may be a reason that forces a firm to practice advance sale especially when unused capacity has no salvage value (Lee and $\mathrm{Ng} 2001$ ).

\subsection{Demand uncertainty}

Demand uncertainty comes from difficulties in predicting customer needs and wants in a given period of time. Information variation across the supply chain is usually greater if the supplier is farther away from the customer. This phenomenon, often referred to as "bullwhip effect" is commonly pronounced in an uncoordinated supply chain network (Chopra and Meindl 2008). Costs associated with bullwhip effects can be as high as 12 to 25 percent for each member in the supply chain (Fawcett et al. 2007). Demand variability can be reduced by application of more advanced forecasting techniques, providing advanced information or smoothing its product consumption through coordination (Bayraktar et al. 2008). However, although both supplier and the customer benefit from these activities the costs associated with them are usually born only by customers. Thus, the need for the supplier to share the costs of the 
activities with the customers or provide other incentives such as price discounts (Kunnumkal and Topaloglu, 2008).

\subsection{Coordination in the supply chain}

Coordination refers to the coordination of diverse elements into an integrated and harmonious operation. Coordination is realized when a decision maker in the supply chain, acting rationally, makes decisions that are efficient for the supply chain as a whole (Gupta and Weerawat, 2006). Companies forming a supply chain are dependent on the performance of other organizations. The need to manage dependencies and different resource flows is important for a company's success (Danese et al. 2004; Patnayakuni et al. 2006). Supply chain coordination is a vehicle for redesigning decision rights, process flow, and resources between supply chain members to influence improved performance (Lee, 2000). Good coordination in the supply chain reduces uncertainty in processing networks (Simatupang et al. 2004), which in turn translates into reduced variability. Some authors argue that coping with uncertainty is the primary motivation for supply chain coordination (Simatupang, et al. 2004). Supply chain coordination offers a means to understand and analyze a supply chain as a set of dependencies. These dependencies exist both in physical flow, which is the flow and storage of goods, and informational flow, which deals with the storage and flow of information associated with those goods (Lewis and Talalayevski, 2004).

Coordination mechanisms provide tools for effectively managing interactions between people, processes, and entities that interact in order to execute supply chain objectives (Xu and Beamon, 2006). They are specific tools designed to address particular coordination problems (Fugate et al. 2006). According to Li and Wang (2007), an important supply chain coordination-mechanism is an operational plan to coordinate the operations of individual supply chain members and improve system profit.

\subsection{Small and medium enterprises}

A number of literature reports the definition of SMEs base on different criteria. Verhees and Meulenberg (2004) define SMEs as a firm that is run and controlled under the direct supervision of the owner. SMEs have also been defined by the number of employees as well as capital investment (Ministry of Industry and Trade, Tanzania, 2002). In recent year SMEs have come under the center of public policy attention (Schlogl, 2004) because of the contribution it offers in the countries' economic growth and job creation. However, in terms of adopting supply chain management (SCM) concept, SMEs tends to be more reluctant mainly because of lack of resources and skills (Verhees and Meulenberg, 2004). Even though the literatures emphasize that SMEs have poor specialization skills, the recent trend indicate the increasing awareness of the importance of key words such as knowledge, skills or competencies for assuring their competitiveness. Most of SMEs operate in a decentralized system where each business entity in the network tries to make decisions which meet individual objectives rather than the entire network. In the case where the enterprise is owned by individual entrepreneur, the choice to adopt or not to adopt a marketing strategy lies on what the owner think about the consequences of the adoption of such strategy in their organization. On the other hand, SMEs today are part of supply chains that require them to manage their processes, orders and participate in collaborative planning and communication to meet local and global competition as a whole.

\section{A case study insight}

The focus study addressed here come from an established small local factory, drying different products of fruits and vegetables in Tanzania. To gain a deep understanding of the factory's business operation, six semi-structured face to face interviews with employees of this factory were conducted to probe information relevant to this study. The respondents provided initial insight into the historical development of the factory. Quantitative and qualitative information for this study were accessible through factory's staffs from production and sales departments.

\subsection{Availability of primary raw materials}

Availability of raw materials are scattered through out of the year but with relatively short harvesting period as shown in Table 2. Production is continuously done by succession of the crop in season. Once two or more crops are in the same season, then production planning, scheduling and rescheduling is done to accommodate the required processes. The products reach ultimate consumers through multiple independent large and small retailers with varying and uncorrelated probability distributions of demand. The relationship which exists between supply chain partners is buy-sell relationship. Each entity is responsible and subjected to the loss which may result from expired products or any other cause.

(Insert Table 2 here)

\subsection{Production technology}

The factory use hybrid solar drier with limited production capacity. The machine is provided with supplementary heating unit to allow continuous process. The technology gives an opportunity for the processor to use solar energy during sunny days and diesel fuel during rain season or night if the process has to continue. Production processing time for each product varies according to water content in the raw materials. Vegetables have less water content as compared to fruits and therefore drying time is short as well. Production cost per unit increases once employing diesel in the 
production process.

Lack of appropriate storage facilities the manufacture keeps limited raw materials to be processed before spoilage starts. Since the product produced has a shelf life of one year, then manufacturer produce enough to meet anticipated market demand. During harvest and production season, the rate of inventory increase for each product is higher than the rate at which the inventory is used up vis-à-vis.

\subsection{Demand for Dried fruits and vegetables}

The three years average data of production, supply and demand of Sonchus spieces and sweet potato leaves are reflected in Figure 1 and Figure 2 respectively. Production of dries Sonchus is carried out in four months of each year; this is because Sonchus spieces grow naturally during rain season. During this season, the factory produces enough products to meet the expected demand of the whole year. The firm forecasts the demand based on the previous year's sales.

(Insert Figure 1 here)

The demand of dried Sonchus and sweet potato leaves fluctuates depending on the season of the year. The processor experience low demand during harvest season because some of consumers prefer to eat freshly harvested vegetables rather than consuming dried vegetable products. The demand start to increase when freshly harvested vegetables becomes scarce and this happen during dry season. Since there is no coordinating mechanisms which exist among the supply chain partners, in some months the supply seems to be higher than the demand and some months supply is low than what the market needs. The implication of this observation is that, in the case where the supply exceeds the demand then, it is likely that one or all of the supply chain partners may remain with large quantity of products at the end of the selling season. Selling season for food products ends when the product expires and there is no salvage value at the end of the selling season. But also, customers become dissatisfied once they go out without the product because of stock out. It should be clear that, dissatisfied customers may not only talk negatively about the firm to other customers but also they are likely to opt for a new supplier or go for an alternative product. For potential customers "word of mouth" is a source of information as most of potential customers tend to trust more non paid agents (Nasution and Mavondo, 2008). It is necessary for the supplier to balance between supply and demand by providing customers with the right quantity at the right place and on time.

(Insert Figure 2 here)

Production of sweet potato leaves takes place in several months more than Sonchus spicies, this is because the crop is drought stable and some farmers irrigate their vegetable gardens to ensure sustainable supply of sweet potato leaves to the market. There is no production towards the end of the year for the reason that, during these months the drought condition is severe enough not to be able to support irrigation activities.

According to Chung et al, (2008) preseason demand information is usually very poor and therefore production levels that exceed or undershoot demand are common. This can be seen from the figures above where the supply sometimes exceeds or do not meet the market demand. This situation may occur as the result of poor forecasting techniques. The demand forecast technique used in this factory is by guessing based on the previous sales data. Bad guessing may cost the factory in terms of time and money including losing potential customers whenever their demand is not well met. The processors tend to produce and keep a substantial amount of safety stock to avoid shortages; on the other hand, retailers are willing to keep low quantity to avoid unnecessary inventory (Frascatore and Mahmoodi, 2008) in such conflicting objectives it creates difficulties in harmonizing the entire network. The factory plans the level and timing of demand to customers individually, resulting in an endless of inventory excess or out of stocks as opposed to collaborative planning (Bowesox et al. 2006), their supply is basically based on push strategy where production and distribution decisions are based on long term forecast (Simchi-Levi et al. 2003).

Delivery of the right quantity, to the right location and at the right time is crucial to the success of any businesses. From Figure 1 and Figure 2 one can notice on how inefficiency delivery can costs the firm, the time in which the product is highly needed no enough supply and the time in which the demand is low there is an excess supply. Since the demand of the products is seasonal, increasing replenishment lead times can contribute to the above observation because the product may enter into another cycle of the demand. Mismatching of production and supply is the result of poor coordination, which in turn translates into an increased demand uncertainty as well as difficulties in managing the demand.

\subsection{Demand trend}

Extrapolation of the three years (36 months) demand data by static method described by Chopra and Meindl (2008) show a seasonal demand cycle, increasing before and after harvesting season. The demand pattern for each processed product repeats year after year (Figure 3 and 4) with two seasons of high demand and one season of low demand i.e. pre-harvest, post harvest and harvesting period respectively. Since the processor is far from the final consumers, it is not easy to have accurate demand data which would help to respond accordingly. 
(Insert figure 3 here)

(Insert figure 4 here)

It would be easy for the processor to predict future demand if the product show a slow demand change (see the slope of a deseasonalized demand) season after season. This happens when fluctuations are considered to be absent. In the real sense the demand for these products are influenced by season i.e. season with high demand and season with low demand. Knowing that trend, the challenge for the processor remain on "how much to produce, where and when to deliver" in respect to the demand trend.

\subsection{Coordinating effect of $A B D$ program on demand uncertainty}

In this section we analyze the coordinating effect of the program on managing demand uncertainty. The reader is reminded to consider the results from the case study as the first option in which each partner strive to optimize individual profit. The study reveals that in some months, the supply overage or underage the demand. The Second option we consider the situation where the processor and the retailer agree to coordinate. The retailer use ABD program as a selling strategy to attract consumers. It is important to note that partners agree to coordinate or collaborate if they realize that the benefits obtain exceeds those they get when operating as an individual entity. As presented by Tang et al. (2002), ABD programs requires customers to commit, pay at a discount price and obtain their orders during the regular selling season. As we can recall, food products are different from other items such as seasonal cards, fashion clothes, electronics among others in the sense that, food products are consumed through out the year and no salvage value once the product expires. However, the demand for food products varies depending on the season of the year. Also the demand may changes due to an unexpected events such as September 11, flood, drought, economic crisis, change in consumers' preference etc. We pay attention on demand uncertainties that the processor experiences in different season of the year but difficult to predict customers' needs "where and when".

Normally, the processor and the retailer have different stocks which comprise different product batches and ages which expire in different times $(t)$, where $t=1,2,3 \ldots x$ and $x$ is the maximum product shelf life. For dried vegetables $x=12$ months. Retailer's inventory vector for dried Sonchus species and sweet potato leaves is: $Q_{r}=$ $q_{1}+q_{2}+q_{3}+\ldots q_{x}$ and the demand is satisfied by first in first out (FIFO). $Q_{r}$ includes the demand $Q_{c} \quad$ for pre-committed customers and demand $Q_{u}$ for uncommitted customers. $Q_{r}$ is expected to be distributed among the three seasons. Which means $Q_{c}=q_{c 1}+q_{c 2}+q_{c 3}$ and $Q_{u}=q_{u 1}+q_{u 2}+q_{u 3}$ where $q_{c}$ and $q_{u}$ are committed and uncommitted quantities respectively, while 1,2, and 3 represent different seasons. The retailer expects the processor to deliver the right quantities in each season to fulfill the committed orders as well as to meet the forecasted demand for uncommitted cgnsumers. On the other hand, the processor's inventory vector for dried Sonchus species and sweet potato leaves is: $Q_{f}=q_{f 1}+q_{f 2}+q_{f 3} . . q_{f m}$ and quantity $Q_{f}$ is expected to meet the processor' predicted demand for the whole year.

Also it should be recalled that the production process activities takes place in a limited period of time i.e. three months for Sonchus species and nine months for sweet potato leaves. Since all products have a limited shelf life of one year, then the product produced in each month expires in the same month of the next year. For example the quantity $Q_{s}$ of Sonchus species produced in April expires in April of the following year. Similarly for quantity $Q_{p}$ of sweet potato leaves. To minimize expired products the quantity $Q_{s}$ and $Q_{p}$ produced must not exceed the total market demand $Q_{m}$ but more important customers' demand have to be met. Theoretically, to operate efficiently and zero expired product while satisfying market demand quantity $Q_{m}$ must be equal to quantity $Q_{s}+Q_{p}$. Apart from distribution factor, to match exactly the production side and demand side in the food industry sector is real difficult and an ideal event. In real life, it is either a certain degree of unfulfilled demand occurs or a certain degree of unused product remains. This happens because forecasts never be perfect. Therefore, an important strategy is to minimize as much as possible the cumulative differences between production and market demand. In this case $\pm \Delta Q=Q_{m}-\left(Q_{s}+Q_{p}\right)$. Uncertainties and errors on predicting demand and production increases when coordination among the supply chain partners does not exist. For example if the retailer predicts to order and sell quantity $Q_{r}$ during the selling seasons, then the question is "does the quantity predicted match with quantity $Q_{f}$ planned and produced by the processor?" This would be possible if both partners agree upon information sharing. ABD program require customers to pre-commit and pay their orders, in such situation portion of the demand $\left(Q_{c}\right)$ is made known and therefore providing useful information in developing more accurate forecasts and distribution plans. It is true that the variance of the demand that occurs in unconditioned purchase is greater than the variance that occurs in a conditioned purchase (pre-committed orders). In conditioned purchases, quantity $q_{c}$ of each season is well known, only quantity $q_{u}$ is uncertain. Since the retailer is close to ultimate consumers, it is also obvious that the retailer has superior demand information than the processor. This means demand error $\left( \pm \Delta q_{u}\right)$ at the retailer's level is much less than the demand error that would happen at the processor's level. If this message with known demand and probably with low demand error of uncertainty goes back to the processor, then the processor will be able to achieve efficient planning of production and distribution among the selling seasons. If the food processing factory addressed in this paper as a case study was working with 
retailers as a coordinated team then all figures would have assumed different shapes that depict more accuracy between production, supply, and demand in their respective seasons.

In general the overall implication of pre-committed order is the decreased demand uncertainty and the best way for SMEs who face financial constraints. It may be a challenging strategy when advanced technologies (electronic data interchange and software) are required to transfer the information. But affordable alternative to electronic data interchange (EDI) and software e.g. webEDI and e-mailing excel spread sheets with demand and inventory information are highly recommended for SMEs.

It should be emphasized that implementation objectives of $\mathrm{ABD}$ program may be the same in different industries but implementation strategies may differ. The nature of the product or service offered by the firm plays a major role in the differences of implementing the program. Within the food industry sector still the difference may be observed. This is because the firm may produce a wide range of products that requires different distribution chain or management.

\subsection{Practical limitations and obstacles of setting up ABD program in SMEs}

It can be recalled that retailers are found into two groups; small and large scale retail traders. The two groups in the food sector differ in many ways including the area which they cover, the number of employees, capital investment and sometimes education level of the owners. Small scale retail shops are scattered in large areas, trading in nearby localities. Usually small scale retail businesses are owned by individual or family members. In the situation where educational level of the owner is low, adoption of new strategy may not be easily acquired. This is because of the fear that information shared may leak and get utilized by rivals to sabotage his business. Information sharing is a key element for successfulness of ABD program as such advanced technologies are needed to convey information efficiently. Apart from capital constraints, lack of technical knowledge may limit the use of advanced technologies in SMEs. Last but not least widely scattered retails shops may impose difficulties in implementation and collection of demand information.

\section{Conclusion}

In this paper an attempt has been made to address the coordinating effects of ABD program in managing demand uncertainty in SMEs food processing industries. A theoretical expression supported by explanatory mathematical equations has been offered. It is noted that supply chain efficiency and reduced demand uncertainty can be achieved once the processor and the retailer agree upon information sharing. This is, of course true and it requires flexible information that support decentralized coordination process.

The growth and sustainable operation of SMEs food processors relies on the performance and efficiency not only in the production side but also in the distribution network. As literatures reports on inventory, through sharing demand and inventory information the visibility in the supply chain increases thus reducing uncertainty of demand of demanded quantities. The increased awareness of demanded quantities will lead to reduced shortage and spoilage costs and, hence, increased profits.

Although theoretical expression show a significant reduced demand uncertainty, we believe that still a lot need to be done in developing coordinating mechanisms of ABD program suitable for SMEs and their specific type of products they produce. This is because processed food products display some uniqueness characteristics and requirements for example shelf life, texture, taste, distribution requirements (normal or cold chain requirements) and processing technologies. Furthermore, we need to extend our research findings to reach stakeholders, influence their decisions, and helping them to create new structures and processes that would help to manage demand uncertainty more efficiently.

We have showed that shared information under ABD program can help the processor to improve the decision towards production planning and distribution. As such incentive to motivate the retailer to share information and means of information monitoring and transfer need to be addressed.

\section{References}

Bayraktar, E., Koh, S.C.L.,Sari, K. \& Tatoglu, E. (2008). The role of forecasting on bullwhip effect for E-SCM applications. International Journal Production Economics, 113, 193-204.

Burke, G.J., Carrillo, J., \& Vakharia, A.J. (2008). Heuristics for sourcing from multiple suppliers with alternative quantity discounts. European Journal of Operational Research, 186, 317-329.

Chopra, S., \& Meindl, P. (2008). Supply chain management. Strategy, planning, and operation. $3^{\text {rd }}$ Ed. Pearson Eductaion Education Asia Ltd, Tsingua University.

Chung, C. Flynn, J. \& Kirca, Ö. (2008). A mult-item newsvendor problem with preseason production and capacitated reactive production. European Journal of Operational Research, 188, 775-792.

Crama, Y., Pascual, R. J., \& Torres, A. (2004). Optimal procurement decisions in the presence of total quantity discounts and alternative product recipes. European Journal of Operational Research, 159, 364-378. 
Danese, P., Romano, P., \& Vinelli, A. (2004). Managing business processes across supply networks: The role of coordination mechanisms. Journal of Purchasing and Supply Management, 10 (4-5), 165-177.

Fawcett, S.E, Ellram, L.M., \& Ogden, J.A. (2007). Supply chain management from vision to implementation.Tsinghua University press.

Frascatore, M.R., \& Mahmoodi, F. (2008). Long-term and penalty contracts in a two-stage supply chain with stochastic demand. European Journal of Operational Research, 184, 147-156.

Fugate, B., Sahin, F., \& Mentzer, J.T. (2006). Supply chain management coordination mechanisms. Journal of Business Logistics, 27 (2), 129-161.

Gupta, D., \& Weerawat, W. (2006). Supplier-manufacturer coordination in capacitated twostage supply chains. European Journal of Operational Research, 175 (1), 67-89.

Kunnumkal, S., \& Topaloglu, H. (2008). Price discounts in exchange for reduced customer demand variability and applications to advance demand information acquisition. International Journal of Production Economics, 111, 543-561.

Lau, A.H.L., Lau, H.S., \& Wang, J.C. (2007). Designing a quantity discount scheme for a newsvendor-type product with numerous heterogeneous retailers. European Journal of Operational Research, 180, 585-600.

Lau, A.H.L., Lau, H.S., \& Zhou, Y.w. (2008). Quantity discount and handling-charge reduction schemes for a manufacturer supplying numerous heterogeneous retailers. Int. J. Production Economic, 113, 425-445.

Lee, H.L. (2000). Creating value through supply chain integration. Supply Chain Management Review, 4 (4), 30-36.

Lee, K.S., \& Ng, I.C.L. (2001). Advanced sale of service capacities: A theoretical analysis of the impact of price sensitivity on pricing and allocations. Journal of Business Research, 54, 219-225.

Lewis I., \& Talalayevsky, A. (2004). Improving the interorganisational supply through optimization of information flows. The Journal of Enterprise Information Management, 17 (3), 229-237.

Li, J., \& Liu, L. (2006). Supply chain coordination with quantity discount policy. International Journal of Production Economics, 101, 89-98.

Li, X., \& Wang, Q. (2007). Coordination mechanisms of supply chain systems. European Journal of Operational Research, 179 (1), 1-16.

Mirmohammadi, S.H., Shadrokh, S., \& Kianfar, F. (2009). An efficient optimal algorithm for the quantity discount problem in material requirement planning. Computers \& Operations Research, 36, 1780-1788.

Nasution, H., N., \& Mavondo, F.T. (2008). Customer value in the hotel industry: What managers believe they deliver and what customer experience. Intertnational Journal of Hospitality Management. 27, 204-213.

Patnayakuni, R., Rai, A., \& Seth, N. (2006). Relational antecedents of information flow integration for supply chain coordination. Journal of Management Informational Systems, 23 (1), 13-49.

Schotanus, F., Telgen, J., \& Boer, L. (2009). Unraveling quantity discount. Omega, 37, 510-521.

Schwart, Z. (2006). Advanced booking and revenue management: Room rates and the consumers' strategic zones. Hospitality Management, 25, 447-462.

Simatupang, T.M., Wright, A.C., \& Sridharan, R. (2002). The knowledge of coordination for supply chain integration. Business Process Management Journal, 8(3), 289-308.

Simchi-Levi, D., Kaminsky, P., \& Simchi-Levi, E. (2003). Designing and managing the supply chain: concepts, strategies and case studies. $2^{\text {nd }}$ Ed. McGraw Hill, Singapore.

Schlogl, H. (2004). Small and medium enterprises: seizing the potential. Observer, 243, 46-8.

Tang, C.S., Rajaram, K., \& Ou, J. (2002). Managing demand uncertainty for short life cycle products using advanced booking discount program. In supply chain management: Methods, applications and research directions. Kluwer Academic Publisher. Netherlands. 69-95.

United Republic of Tanzania, Ministry of Industry and Trade. (2002). Small and Medium Enterprises Development Policy.

Verhees, J.H.M. Frans and Matthew T.G. Meulenberg. (2004), Market Orientation, Innovativeness, Product Innovation, and Performance in Small Firms. Journal of Small Business Management, 42 (2), 134-154.

Xu, L., \& Beamon, B.M. (2006). Supply chain coordination and cooperation mechanisms: An attribute-based approach. The Journal of Supply Chain Management, 42 (1), 4-12. 
Table 1. Harvesting season for fruits and vegetable

\begin{tabular}{|c|c|}
\hline Raw material & Available \\
\hline Mangoes & December-January and April-July \\
\hline Jackfruit & October-December \\
\hline Oranges & April-July \\
\hline Sweet potato leaves & January-September \\
\hline Sonchus species & April-July \\
\hline
\end{tabular}

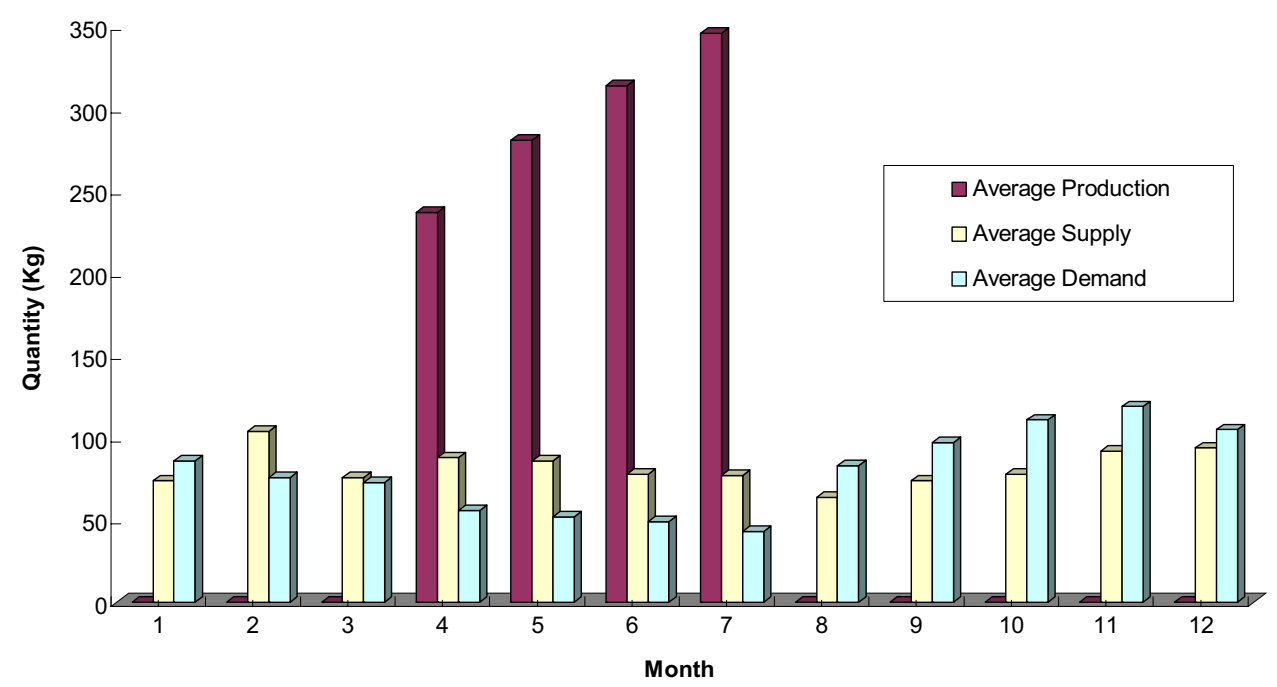

Figure 1: Dried Sonchus species

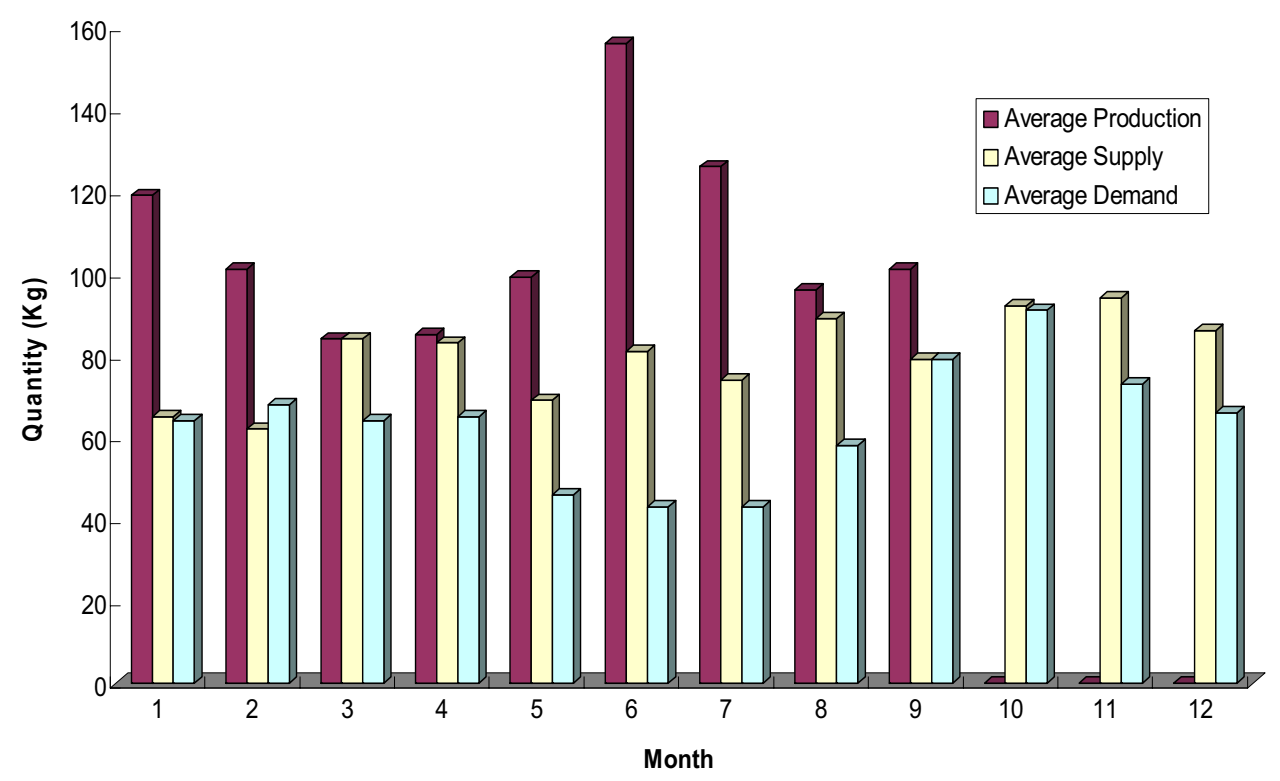

Figure 2: Dried sweet potato leaves 


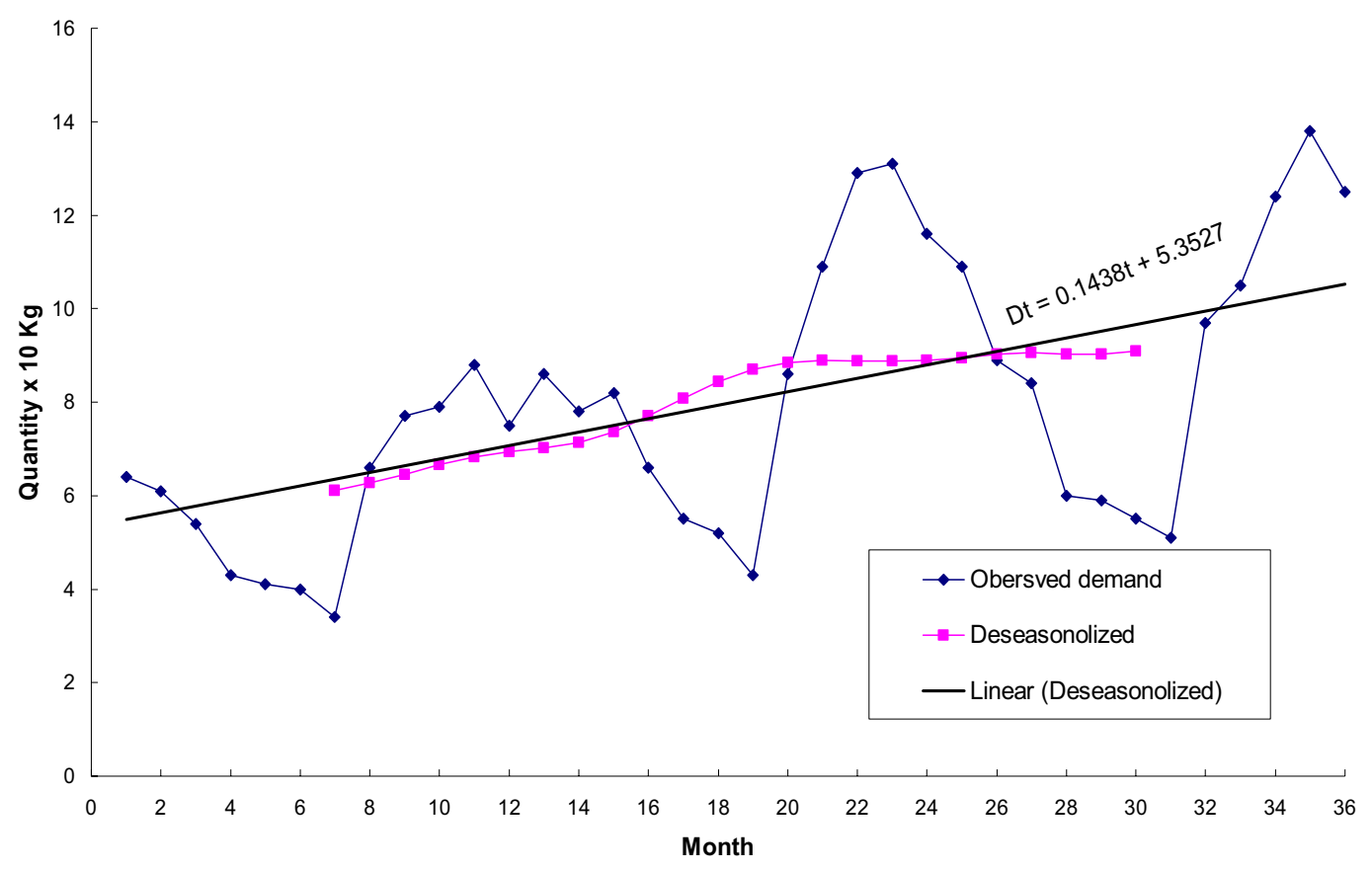

Figure 3: Observed and deseasonalized demand for Sonchus spp

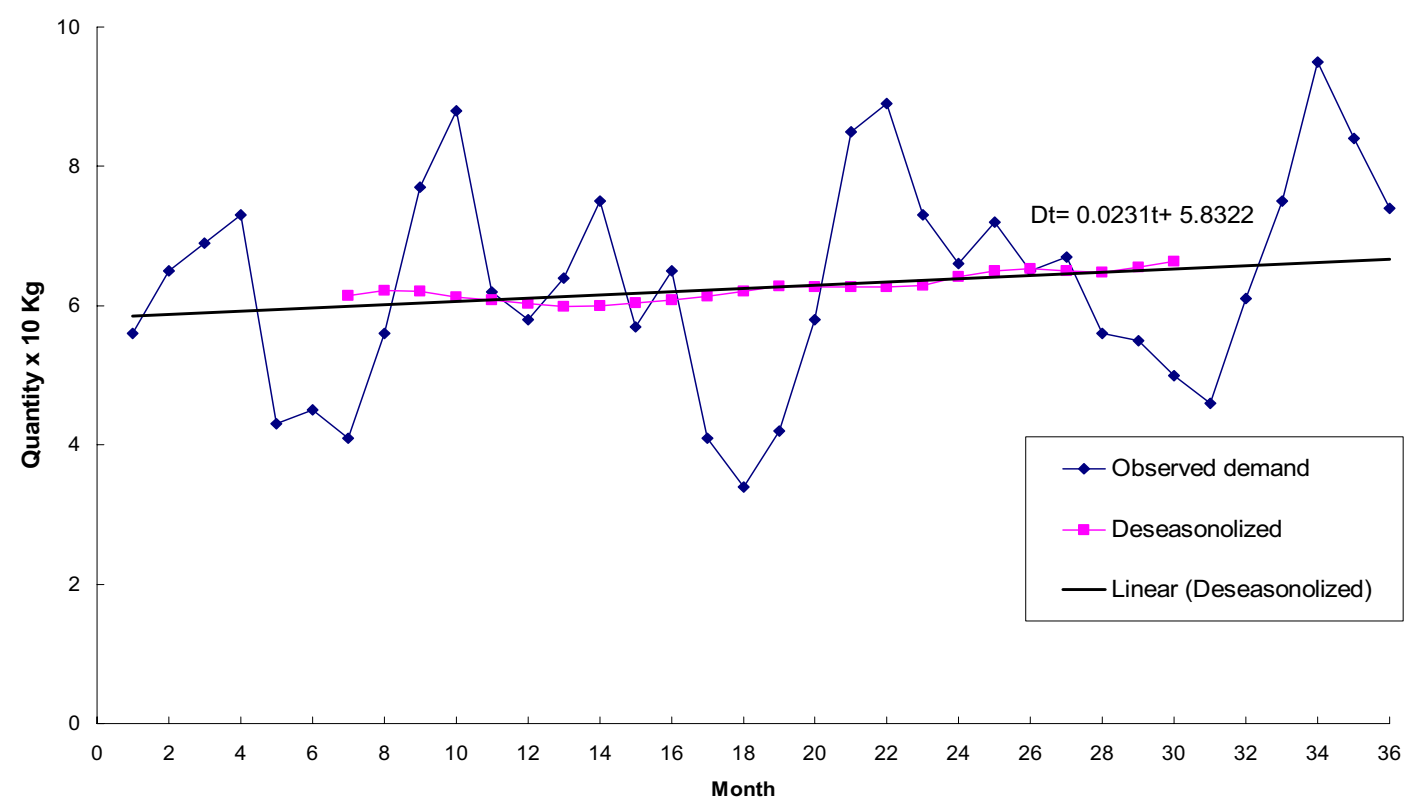

Figure 4: Observed and deseasonalized demand for sweet potato leaves 